Bull. Korean Math. Soc. 49 (2012), No. 1, pp. 63-74

http://dx.doi.org/10.4134/BKMS.2012.49.1.063

\title{
RECONSTRUCTION THEOREM FOR STATIONARY MONOTONE QUANTUM MARKOV PROCESSES*
}

\author{
Jaeseong Heo ${ }^{\dagger}$, Viacheslav P. Belavkin, and Un Cig JI ${ }^{\ddagger}$ \\ Abstract. Based on the Hilbert $C^{*}$-module structure we study the re- \\ construction theorem for stationary monotone quantum Markov processes \\ from quantum dynamical semigroups. We prove that the quantum sto- \\ chastic monotone process constructed from a covariant quantum dynam- \\ ical semigroup is again covariant in the strong sense.
}

\section{Introduction}

A quantum dynamical semigroup as a quantum analogue of a classical stationary Markov evolution provides a mathematical framework for the study of dissipative dynamics in quantum open system. The mathematical structure of quantum dynamical semigroups and their generators have been studied by many authors $[7,9,15]$ and the references therein.

The notion of a weak quantum Markov process in terms of stochastic representation for a quantum dynamical semigroup by a classical stochastic process of counting type in continuous time $T=\mathbb{R}_{+}$was first introduced by Davies in [8]. Since then, as a generalization of the Kolmogorov construction for the classical case to the quantum case, the reconstruction theorem for a quantum stochastic process in the weak sense and its classical stochastic representation have been studied by several authors. The reconstructions of quantum Markov processes in strong sense were studied in discrete time $T=\mathbb{Z}_{+}$by Lindblad in [16], and Belavkin [2,3] generalized that for a quantum non-stationary and non-Markov process indexed by an arbitrary causally-ordered space-time $T$ to

Received April 30, 2010; Revised August 24, 2010.

2010 Mathematics Subject Classification. Primary 60G20, 46L08; Secondary 60G10, $46 \mathrm{~L} 55$.

Key words and phrases. Hilbert $C^{*}$-module, covariant representation, quantum stochastic process, quantum dynamical semigroup.

* This joint work was supported by PMI2 project of UK-Korea collaborations.

$\dagger$ This research was supported by Basic Science Research Program through the National Research Foundation of Korea (NRF) funded by MEST (2010-0008447).

$¥$ This work was partially supported by the Korea Science and Engineering Foundation (KOSEF) grant funded by the Korea government (MEST) (No. R01-2008-000-10843-0). 
include also the classical stochastic representation of Davies type. Independently, Accardi-Frigerio-Lewis in [1] considered the reconstruction of a nonMarkov quantum stochastic process in strong sense which does not require any order in $T$ but requires not only casually ordered but also noncausal correlation kernels forming an infinite set for a finite $T$. On the other hand, Lindblad, Belavkin and later Parthasarathy [17] studied the monotone reconstruction theorem for a weak quantum stochastic process using only causal correlation kernels which form a finite set for a finite $T$. Given the kernels by a quantum dynamical semigroup, this monotone reconstruction is a dynamical versions of Stinespring's theorem respectively in discrete and continuous time $T$.

The main purpose of this paper is to study the Belavkin reconstruction theorem [2] for monotone covariant quantum stochastic Markov processes from the point of view of the Hilbert $C^{*}$-module structure. Such process is given in weak sense by a positive semi-definite function constructed from a quantum dynamical semigroup, and we prove that the strong monotone covariant quantum stochastic process reconstructed from a covariant positive semi-definite function given by a covariant quantum dynamical semigroup is again covariant in strong sense as in [3].

This paper is organized as follows: In Section 2, we recall some basic notions for Hilbert $C^{*}$-modules. In Section 3, we study a covariant representation, constructed from a completely positive map, of a $C^{*}$-dynamical system on a symmetry semigroup and the reconstruction theorem for a Hilbert $C^{*}$-module valued stationary stochastic process, see Theorem 3.1. In Section 4 , we study the reconstruction theorem for monotone quantum stochastic processes from quantum dynamical semigroups, see Theorem 4.1 , which is a $C^{*}$-generalization of Parthasarathy's construction in [17] and for the stationary semigroup case of the constructions given by Belavkin $[2,3]$. In Section 5 , we prove that the monotone quantum stochastic process constructed from a covariant quantum dynamical semigroup is strongly covariant with respect to a certain unitary representation of the given symmetry semigroup.

\section{Hilbert $C^{*}$-modules}

Let $\mathcal{A}$ be a $C^{*}$-algebra. A right $\mathcal{A}$-module $\mathfrak{X}$ is called a pre-Hilbert $\mathcal{A}$ module if there is an $\mathcal{A}$-valued inner product $\langle x \mid z\rangle_{\mathfrak{X}} \equiv\langle x \mid z\rangle$ as a mapping $\langle\cdot \mid \cdot\rangle: \mathfrak{X} \times \mathfrak{X} \rightarrow \mathcal{A}$ which is linear in the second variable and satisfies the following properties:

(i) $|x|^{2}:=\langle x \mid x\rangle \geq 0$, and $|x|^{2}=0$ only if $x=0$,

(ii) $\langle x \mid z\rangle=\langle z \mid x\rangle^{*}$ for any $x, z \in \mathfrak{X}$,

(iii) $\langle x \mid z a\rangle=\langle x \mid z\rangle a$ for any $a \in \mathcal{A}$.

If, in addition, $\mathfrak{X}$ is complete with respect to the norm $\|x\|=\|\langle x \mid x\rangle\|^{1 / 2}$, then $\mathfrak{X}$ is called a $($ right $)$ Hilbert $\mathcal{A}$-module. Obviously, $\mathfrak{X}=\mathcal{A}$ is the right Hilbert $\mathcal{A}$ module with respect to $\langle a \mid b\rangle=a^{*} b$ such that the left multiplication operator $L_{a} b=a b$ on $\mathcal{A}$ has the adjoint $L_{a}^{\dagger}=L_{a^{*}}$. Note that in general the vectors 
$x \in \mathfrak{X}$ are in one-to-one correspondence with the linear operators $L_{x}: \mathcal{A} \rightarrow \mathfrak{X}$ of the left multiplications $L_{x} a=x a$ given by the right action $a: x \mapsto x a$ of $a$ on $\mathfrak{X}$ due to the existence of an approximate identity in $\mathcal{A}$. This correspondence is explicit: $x 1=x=L_{x} 1$ in the case of the unital $C^{*}$-algebra $\mathcal{A} \ni 1$, so we might not even distinguish $x$ and $L_{x}$.

Let $\mathfrak{X}$ and $\mathfrak{Y}$ be Hilbert $\mathcal{A}$-modules. We denote by $\mathcal{L}_{\mathcal{A}}(\mathfrak{X}, \mathfrak{Y})$ the space of adjointable operators mapping $\mathfrak{X}$ into $\mathfrak{Y}$ such that for each $L \in \mathcal{L}_{\mathcal{A}}(\mathfrak{X}, \mathfrak{Y})$ there is an adjoint operator $L^{\dagger} \in \mathcal{L}_{\mathcal{A}}(\mathfrak{Y}, \mathfrak{X})$ in the sense that

$$
\langle L x \mid y\rangle_{\mathfrak{Y}}=\left\langle x \mid L^{\dagger} y\right\rangle_{\mathfrak{X}}, \quad(x \in \mathfrak{X}, y \in \mathfrak{Y}),
$$

and by $\mathcal{B}_{\mathcal{A}}(\mathfrak{X}, \mathfrak{Y})$ the space of all linear bounded operators $L: \mathfrak{X} \rightarrow \mathfrak{Y}$ satisfying the right $\mathcal{A}$-modular property

$$
L(x a)=(L x) a, \quad(x \in \mathfrak{X}, a \in \mathcal{A}) .
$$

It follows from the uniform boundedness theorem that each operator $L \in$ $\mathcal{L}_{\mathcal{A}}(\mathfrak{X}, \mathfrak{Y})$ is bounded, so that $L \in \mathcal{B}_{\mathcal{A}}(\mathfrak{X}, \mathfrak{Y})$. We write $\mathcal{L}_{\mathcal{A}}(\mathfrak{X})$ for $\mathcal{L}_{\mathcal{A}}(\mathfrak{X}, \mathfrak{X})$ which becomes a $C^{*}$-algebra with the operator norm. A representation of a $C^{*}$-algebra $\mathcal{B}$ on a Hilbert $\mathcal{A}$-module $\mathfrak{X}$ is a $*$-homomorphism $\pi$ from $\mathcal{B}$ into $\mathcal{L}_{\mathcal{A}}(\mathfrak{X})$. Note that the elements $x \in \mathfrak{X}$ are adjointable, $x^{\dagger} z=\langle x \mid z\rangle$, as the linear operators $L_{x} \in \mathcal{L}_{\mathcal{A}}(\mathcal{A}, \mathfrak{X})$, as well as $x^{\dagger}:=L_{x}^{\dagger} \in \mathcal{L}_{\mathcal{A}}(\mathfrak{X}, \mathcal{A})$ with $x^{\dagger \dagger}=x \equiv L_{x}$. But the linear operator of the right multiplication $R_{a} x=x a$ on $\mathfrak{X}$ is not adjointable, it is not even $\mathcal{A}$-modular, $R_{a} \notin \mathcal{B}_{\mathcal{A}}(\mathfrak{X})$ unless $\langle\cdot \mid \cdot\rangle$ has the range in a commutative ideal of $\mathcal{A}$, in which case $R_{a}^{\dagger}=R_{a^{*}}$. For more details on Hilbert $C^{*}$-modules, we refer to [14].

\section{3. $C^{*}$-dynamical systems and stochastic processes}

Let $\mathcal{A}$ be a unital $C^{*}$-algebra and let $\left(u_{s}\right):=\left\{u_{s} \in \mathcal{U}(\mathcal{A}): s \in S\right\}$ be a representation of a symmetry semigroup $S$ in the unitary group $\mathcal{U}(\mathcal{A})$. Let $\theta$ be an action of $S$ on a (unital) $C^{*}$-algebra $\mathcal{B}$ by a semigroup of endomorphisms $s \mapsto \theta_{s} \in \operatorname{End}(\mathcal{B})$ such that for each $s \in S$ the map $\theta_{s}: \mathcal{B} \rightarrow \mathcal{B}$ is an injective endomorphism (shift) on $\mathcal{B}$, and $\theta_{s} \circ \theta_{r}=\theta_{s . r}$. The triple $(\mathcal{B}, S, \theta)$ is called a $C^{*}$-dynamical system.

A linear map $\phi: \mathcal{B} \rightarrow \mathcal{A}$ is called $(\theta, u)$-covariant if

$$
\phi\left(\theta_{s}(b)\right)=u_{s} \phi(b) u_{s}^{*} \quad \text { for all } b \in \mathcal{B}, s \in S .
$$

A covariant representation of $(\mathcal{B}, S, \theta)$ is a triple $(\pi, V, \mathfrak{X})$, where $\pi$ is a (unital) *-homomorphism of $\mathcal{B}$ on a Hilbert $\mathcal{A}$-module $\mathfrak{X}$ and $V$ is an isometric $\mathcal{A}$ modular map $V: S \rightarrow \mathcal{B}_{\mathcal{A}}(\mathfrak{X})$ of $S$ such that $V_{r} V_{s}=V_{r . s}$ for all $r, s \in S$, $\left|V_{s} x\right|^{2}=|x|^{2}$ for all $x \in \mathfrak{X}$ and

$$
\pi\left(\theta_{s}(b)\right) V_{s}=V_{s} \pi(b), \quad(b \in \mathcal{B}, s \in S) .
$$

If isometries $V_{s} \in \mathcal{B}_{\mathcal{A}}(\mathfrak{X})$ are all surjective, they are obviously adjointable, $V_{s} \in \mathcal{L}_{\mathcal{A}}(\mathfrak{X})$ as being unitary: $V_{s}^{\dagger}=V_{s}^{-1}$. 
Theorem 3.1. Let $(\mathcal{B}, S, \theta)$ be a $C^{*}$-dynamical system with the unit element $1_{\mathcal{B}}$ and let $u$ be a representation of a semigroup $S$ into the unitary group $\mathcal{U}(\mathcal{A})$ of a unital $C^{*}$-algebra $\mathcal{A}$ with the unit element $1_{\mathcal{A}}$. If $\phi: \mathcal{B} \rightarrow \mathcal{A}$ is a unital $(\theta, u)$-covariant completely positive linear map, then

(i) there exists a covariant representation $(\pi, V, \mathfrak{X})$ of $(\mathcal{B}, S, \theta)$ with a unit vector $e \in \mathfrak{X}$ generating $\mathfrak{X}$ by $\pi(\mathcal{B})$ e $\mathcal{A}$ such that $\phi(b)=\langle e \mid \pi(b) e\rangle$,

(ii) for any $\phi$-stationary process $\left\{b_{t}: t \in S\right\} \subset \mathcal{B}$, i.e.,

$$
\phi\left(b_{r \cdot t}^{*} b_{s \cdot t}\right)=\phi\left(b_{r}^{*} b_{s}\right) \quad(r, s, t \in S),
$$

the $\mathfrak{X}$-valued process $\left\{x_{t} \equiv \pi\left(b_{t}\right)\right.$ eu $\left.u_{t}: t \in S\right\}$ is u-stationary, that is,

$$
C_{r \cdot t, s \cdot t}(x)=u_{t}^{*} C_{r, s}(x) u_{t} \quad \text { for all } r, s, t \in S,
$$

in particular, $\left\{V_{t} e: t \in S\right\}$ is u-stationary, where

$$
C_{r, s}(x)=\left\langle x_{r} \mid x_{s}\right\rangle \text {. }
$$

Proof. (i) By similar arguments to the Paschke's construction [18, 11], there exist a Hilbert $\mathcal{A}$-module $\mathfrak{X}$ and a $*$-representation $\pi: \mathcal{B} \rightarrow \mathcal{L}_{\mathcal{A}}(\mathfrak{X})$. In fact, $\mathfrak{X}$ is the completion of the quotient space $\mathcal{B} \otimes_{\text {alg }} \mathcal{A} / \mathcal{N}$ by the pre-inner product $\langle\cdot \mid \cdot\rangle$ defined by

$$
\left\langle\sum_{i} b_{i} \otimes a_{i} \mid \sum_{j} b_{j}^{\prime} \otimes a_{j}^{\prime}\right\rangle=\sum_{i, j} a_{i}^{*} \phi\left(b_{i}^{*} b_{j}^{\prime}\right) a_{j}^{\prime}
$$

on the algebraic tensor product $\mathcal{B} \otimes_{\text {alg }} \mathcal{A}$, where $\mathcal{N}=\left\{z \in \mathcal{B} \otimes_{\text {alg }} \mathcal{A}:\langle z \mid z\rangle=\right.$ $0\}$. For each $b \in \mathcal{B}$, the map

$$
\pi(b): \sum_{i} b_{i} \otimes a_{i} \longmapsto \sum_{i} b b_{i} \otimes a_{i}
$$

can be extended to $\mathfrak{X}$ by continuity, which gives the $*$-representation $\pi$. In this construction, the unit vector $e=1_{\mathcal{B}} \otimes 1_{\mathcal{A}} \in \mathfrak{X}$ is a generating vector of $\mathfrak{X}$, i.e., $\pi(\mathcal{B}) e \mathcal{A}=\{\pi(b) e a \mid a \in \mathcal{A}, b \in \mathcal{B}\}$ spans $\mathfrak{X}$, and we have $\phi(b)=\langle e \mid \pi(b) e\rangle$ for any $b \in \mathcal{B}$. On the other hand, for any $a \in \mathcal{A}, b \in \mathcal{B}$ and $s \in S$, by the $(\theta, u)$-covariance of $\phi$ we have

$$
\left|\sum_{i} \pi\left(\theta_{s}\left(b_{i}\right)\right) e u_{s} a_{i}\right|^{2}=\left|\sum_{i} \pi\left(b_{i}\right) e a_{i}\right|^{2}
$$

and so the operator

$$
V_{s}: \pi(b) e a \mapsto \pi\left(\theta_{s}(b)\right) e u_{s} a
$$

can be extended to $\mathfrak{X}$ by linearity and continuity. Moreover, $V_{s}$ satisfies the $\mathcal{A}$-modular property. By the definition of $V_{s}$, we have $V_{r} V_{s}=V_{r \cdot s}$ on $\pi(\mathcal{B}) e \mathcal{A}$, which also holds on $\mathfrak{X}$. Furthermore, it satisfies the covariance condition (3.1) on $\pi(\mathcal{B}) e \mathcal{A}$ which also holds by continuity on the whole space $\mathfrak{X}$. Therefore, the triple $(\pi, V, \mathfrak{X})$ is a covariant representation of $(\mathcal{B}, S, \theta)$. 
(ii) The proof of the stationary condition (3.2) is straightforward from the definition of the covariance function $C_{r, s}(x)$ and the $\phi$-stationarity of $\left\{b_{t}: t \in\right.$ $S\}$.

Remark 3.2. In (i) of Theorem 3.1, a covariant representation $(\pi, V, \mathfrak{X})$ of $(\mathcal{B}, S, \theta)$ is said to be minimal if there exists a unit vector $e \in \mathfrak{X}$ such that $\pi(\mathcal{B}) e \mathcal{A}$ spans a dense subspace of $\mathfrak{X}$. A minimal covariant representation is unique up to unitary equivalence. That is, if there exists another minimal covariant representation $\left(\pi^{\prime}, V^{\prime}, \mathfrak{X}^{\prime}\right)$ of $(\mathcal{B}, S, \theta)$ with a generating vector $e^{\prime} \in \mathfrak{X}^{\prime}$, then the map $U: \mathfrak{X} \rightarrow \mathfrak{X}^{\prime}$ given by

$$
U(\pi(b) e a)=\pi^{\prime}(b) e^{\prime} a, \quad(b \in \mathcal{B}, a \in \mathcal{A})
$$

gives the unitary equivalence of the covariance representations. In this case, $U V U^{\dagger}$ coincides with the isometric representation $V^{\prime}$ of $S$ on $\mathfrak{X}^{\prime}$.

In (ii) of Theorem 3.1, for each $b \in \mathcal{B}$ such that

$$
\phi\left(\theta_{r \cdot t}(b)^{*} \theta_{s \cdot t}(b)\right)=\phi\left(\theta_{r}(b)^{*} \theta_{s}(b)\right), \quad(r, s, t \in S)
$$

the $\mathfrak{X}$-valued process $\left\{x_{t} \equiv V_{t} \pi(b) e=\pi\left(\theta_{t}(b)\right) e u_{t}: t \in S\right\}$ is $u$-stationary.

\section{Quantum dynamical semigroups and Markov $C^{*}$-processes}

Let $T=[0, \infty)$ be an additive semigroup. A quantum dynamical conservative semigroup over a unital $C^{*}$-algebra $\mathcal{A}$ is a one-parameter family $\phi=\left\{\phi_{t}: t \in\right.$ $T\}$ of unital completely positive (UCP) linear maps $\phi_{t}: \mathcal{A} \rightarrow \mathcal{A}$ satisfying the dynamical condition:

$$
\phi_{s} \circ \phi_{t}=\phi_{s+t}, \quad(s, t \in T)
$$

and is conservative in the sense that $\phi_{t}\left(1_{\mathcal{A}}\right)=1_{\mathcal{A}}$ for all $t \in T$.

In [17], Parthasarathy proved that, if $\phi$ is a dynamical semigroup of normal UCP maps on a von Neumann algebra $\mathcal{M}$ on a Hilbert space $\mathcal{H}_{0}$, then there exist a family of normal unital representations $\left(\pi_{t}, \mathcal{H}_{t}\right)$ of $\mathcal{M}$ with $\pi_{0}=\mathrm{id}_{\mathcal{M}}$ and a family $F$ of Hilbert space co-isometries $F_{s}(t): \mathcal{H}_{t} \rightarrow \mathcal{H}_{s}(s<t)$ satisfying the backward dynamical condition

$$
F_{r}(s) F_{s}(t)=F_{r}(t), \quad(t>s>r \in T)
$$

such that

$$
F_{r}(t) \pi_{t}(a) F_{r}(t)^{\dagger}=\pi_{r}\left(\phi_{t-r}(a)\right) \quad \text { for all } a \in \mathcal{M}, t>r \in T .
$$

This may be considered as a dynamical type Stinespring theorem for a semigroup of normal completely positive maps. One of our main interests in this paper is to give the Hilbert $C^{*}$-module version (Theorem 4.1). For the study of the non-stationary case, we refer to [2,3] by Belavkin.

Let $\left\{\mathfrak{X}_{t}: t \in T\right\}$ be a family of Hilbert $\mathcal{A}$-modules. A family $\left\{F_{r}(t): r<t \in\right.$ $T\}$ of operators $F_{r}(t): \mathfrak{X}_{t} \rightarrow \mathfrak{X}_{r}(r<t)$ is called a hemigroup if the backward dynamical condition (4.2) holds and $F_{r}(r)=I$ for all $r \in T$. 
In [4], Bhat and Parthasarathy proved to show the existence of weak Markov flows dilating a given completely positive semigroup $\left\{T_{t}: t \geq 0\right\}$ in both the conservative case, $T_{t}(1)=1$ and the nonconservative case, $T_{t}(1) \leq 1$. Bhat and Skeide [5] extended a completely positive semigroup on a unital $C^{*}$-subalgebra of $\mathcal{B}(\mathcal{H})$ to an $E_{0}$-semigroup which is a strongly continuous semigroup of unital $*$-endomorphisms on $\mathcal{B}(\mathcal{H})$ by using of continuous families of Hilbert $C^{*}$-modules. In [5, Theorem 6.9] they showed the existence of the correlation kernel of a completely positive semigroup, which is similar to our kernel given in the following theorem. However, we construct a hemigroup of co-isometries and a increasing family of Hilbert $C^{*}$-modules which is different from the one in [5]. Moreover, we will use the proof of Theorem 4.1 to prove Theorem 5.1 which is the covariant version of the following theorem, so that we will give a proof of the following theorem for the reader's convenience.

Theorem 4.1. Let $\left\{\phi_{t}: t \in T\right\}$ be a quantum dynamical conservative semigroup over a unital $C^{*}$-algebra $\mathcal{A}$ with the unit element 1 . Then there exist

(i) an increasing family $\mathfrak{X}=\left\{\mathfrak{X}_{t}: t \in T\right\}$ of Hilbert $\mathcal{A}$-modules,

(ii) a family $\pi=\left\{\pi_{t}: t \in T\right\}$ of *-representations $\pi_{t}$ of $\mathcal{A}$ on $\mathfrak{X}_{t}$ with $\pi_{0}=\mathrm{id}_{\mathcal{A}}$

(iii) a hemigroup $F=\left\{F_{r}(t): r<t \in T\right\}$ of co-isometries $F_{r}(t): \mathfrak{X}_{t} \rightarrow \mathfrak{X}_{r}$ such that

$$
\left|\pi_{t}(a) F_{t}^{\dagger}\left(t_{n}\right) \pi_{t_{n}}\left(a_{n}\right) \cdots F_{t_{2}}^{\dagger}\left(t_{1}\right) \pi_{t_{1}}\left(a_{1}\right) e_{t_{1}}\right|^{2}=\kappa_{\emptyset}^{\tau}\left(\mathbf{a}^{*}, \mathbf{a}\right)
$$

for all $\mathbf{a}=\left(a_{1}, \ldots, a_{n}, a\right) \in \mathcal{A}^{n+1}$ and the state vector-martingale $e_{t}=F_{t}^{\dagger}(0) 1$, where $\kappa_{0}^{\tau}\left(\mathbf{a}^{*}, \mathbf{a}\right)$ is defined as in (4.6). In particular, the process $\pi$ satisfies the strong Markov property

$$
F_{r}(t) \pi_{t}\left(\mathcal{B}_{t}\right) F_{r}(t)^{\dagger} \subseteq \pi_{r}\left(\mathcal{B}_{r}\right) \quad \text { for all } t>r \in T .
$$

Proof. The proof is similar to those of the reconstruction theorems by Belavkin [3] and Parthasarathy [17]. By similar arguments used in the proof of Theorem 3.1 , we obtain a family of the KS-GNS triples $\left(\mathfrak{X}_{t}^{\circ}, \pi_{t}^{\circ}, e_{t}^{\circ}\right)$ from the completely positive linear map $\phi_{t}$ which we now may denote as $\left(\mathfrak{X}_{t}^{\tau}, \pi_{t}^{\tau}, e_{t}^{\tau}\right)$ with $\tau=\{t\}$.

For each finite chain $\tau=\left\{t_{1}, \ldots, t_{n}, t\right\} \subset T$ with $0<t_{1}<\cdots<t_{n}<t$, we define a multilinear map $\kappa_{0}^{\tau}: \mathcal{A}^{n+1} \times \mathcal{A}^{n+1} \rightarrow \mathcal{A}$ indexed by $\tau$ as follows:

$$
\kappa_{0}^{\tau}\left(\mathbf{a}^{*}, \mathbf{b}\right)=\phi_{t_{1}}\left(a_{1}^{*} \phi_{t_{2}-t_{1}}\left(\cdots a_{n}^{*} \phi_{t-t_{n}}\left(a^{*} b\right) b_{n} \cdots\right) b_{1}\right) .
$$

Here $\mathbf{a}^{*}=\left(a_{1}^{*}, \ldots, a_{n}^{*}, a^{*}\right) \in \mathcal{A}^{n+1}$ for any $(n+1)$-tuple $\mathbf{a}=\left(a_{1}, \ldots, a_{n}, a\right) \in$ $\mathcal{A}^{n+1}$. Then each kernel $\kappa_{0}^{\tau}$ is positive semi-definite due to the complete positivity of $\phi$ and satisfies the projectivity (consistency) condition

$$
\kappa_{0}^{\tau^{\prime}}\left(\mathbf{a}_{\tau}^{*}, \mathbf{b}_{\tau}\right)=\kappa_{0}^{\tau}\left(\mathbf{a}^{*}, \mathbf{b}\right), \quad\left(\tau \subset \tau^{\prime}, \mathbf{a}, \mathbf{b} \in \mathcal{A}^{|\tau|}\right)
$$

where $\mathbf{a}_{\tau} \in \mathcal{A}^{\left|\tau^{\prime}\right|}$ is $\mathbf{a} \in \mathcal{A}^{|\tau|}$ injected into $\mathcal{A}^{\left|\tau^{\prime}\right|}$ by adding the identities $a_{k}=$ $1 \in \mathcal{A}$ to a for all $t_{k} \in \tau^{\prime}$ which are not in $\tau$, due to the unitality of $\phi$. The 
kernel $\kappa_{0}^{\tau}$ satisfies the last moment adjointability property:

$$
\kappa_{0}^{\tau}\left(\mathbf{a}^{*}, b_{|\tau|} \cdot \mathbf{b}\right)=\kappa_{0}^{\tau}\left(\mathbf{a}^{*} \cdot b_{|\tau|}, \mathbf{b}\right), \quad\left(\mathbf{a} \in \mathcal{A}^{|\tau|}, b \in \mathcal{A}\right)
$$

where $\mathbf{a}^{*} \cdot b_{|\tau|}=\left(a_{1}^{*}, \ldots, a_{n}^{*}, a^{*} b\right)=\left(b_{|\tau|}^{*} \cdot \mathbf{a}\right)^{*}$ for $\tau=\left\{t_{1}, \ldots, t_{n}, t\right\}$.

Let $\mathcal{F}$ be the set of all functions $f: T \rightarrow \mathcal{A}$ with finite support, i.e., $\operatorname{supp}(f):=\{t \in T \mid f(t) \neq 1\}$ is a finite chain $\tau(f) \subset T$. Let $\mathcal{F}_{t}$ be the subset of $\mathcal{F}$ consisting of $f$ having the support $\tau(f) \subset[0, t]$ with $\max \tau(f) \leq t$. Let $\tau$ be a finite nonempty chain $\tau=\left\{t_{1}, \ldots, t_{n}, t\right\}$ and let $\mathbf{a} \in \mathcal{A}^{|\tau|}$ be a $(n+1)$-tuple $\left(a_{1}, \ldots a_{n}, a\right)$ defining the function $f \in \mathcal{F}_{t}$ with the support $\operatorname{supp}(f) \subset \tau$ by $f\left(t_{i}\right)=a_{i}, f(t)=a$ and $f(0)=1$. Any other function $f \in \mathcal{F}_{t}$ has $f(0)=c \neq 1$ and is defined by $\mathbf{a}_{0}=(c, \mathbf{a})$. We set

$$
C_{t}(f ; h)=c^{*} \kappa_{0}^{\tau}\left(\mathbf{a}^{*}, \mathbf{b}\right) d \equiv \kappa_{[0}^{\tau}\left(\mathbf{a}_{0}^{*}, \mathbf{b}_{0}\right), \quad t=\max \tau
$$

for any $f, h \in \mathcal{F}_{t}$ with $h\left(t_{i}\right)=b_{i} \in \mathcal{A}$ including $h(0)=d \in \mathcal{A}$ and $h(t)=b \in \mathcal{B}_{t}$ and notice that due to the projectivity condition (4.7), the equation (4.9) does not depend on $\tau \leq t$ and therefore on $t$ as soon as $\tau \supseteq(\tau(f) \cup \tau(h)) \backslash\{0\}$ contains the joint support of $f, h \in \mathcal{F}_{t}$ without 0 . The kernel $C(f ; h)=\kappa_{0}^{\tau}\left(f^{*}, h\right)$ extending by polarization the positive values $\kappa\left(f^{*}, f\right) \geq 0$ is also positive semidefinite on $\mathcal{F}_{t}$.

Take the inductive union $\mathcal{F}=\bigcup \mathcal{F}_{t}$ and its $\mathcal{A}$-linear span $\mathcal{F} \mathcal{A}$ as a right $\mathcal{A}$-module of formal $\mathcal{A}$-combinations $g=\sum_{i \in I} f_{i} a_{i}$ with $g a=\sum_{i \in I} f_{i} a_{i} a$. We may regard $g$ as the $\mathcal{A}$-valued function of $\mathcal{F}$ to $\mathcal{A}$ with finite support

$$
\operatorname{supp}(g)=\{f \in \mathcal{F} \mid g(f) \neq 0\}=\left\{f_{i} \mid i \in I\right\}
$$

such that $g\left(f_{i}\right)=a_{i}$. Define an $\mathcal{A}$-valued semi-definite sesquilinear form

$$
\langle g \mid g\rangle=\sum_{i, k} a_{i}^{*} C_{t}\left(f_{i} ; f_{k}\right) a_{k} \equiv \sum_{f, h \in \operatorname{supp}(g)} g(f)^{*} C_{t}(f ; h) g(h)
$$

for any $g \in \mathcal{F}_{t} \mathcal{A}$ having the support in $\mathcal{F}_{t}$. Due to the projectivity of $C$, this does not depend on $t$ as soon as $\operatorname{supp}(g) \subseteq \mathcal{F}_{t}$. Take the quotient $\mathfrak{D}_{t}=$ $\mathcal{F}_{t} \mathcal{A} / \operatorname{ker} C_{t}$ where $\operatorname{ker} C_{t}=\left\{g \in \mathcal{F}_{t} \mathcal{A} \mid\langle g \mid g\rangle=0\right\}$ and complete it. The completion $\mathfrak{X}_{t}$ is the right Hilbert $\mathcal{A}$-module with $x_{t}(h) \in \mathfrak{X}_{t}, h \in \mathcal{F}_{t}$, defined as the equivalence class of the indicator $\delta_{h}$ such that

$$
\left\langle x_{t}(f) \mid x_{t}(h)\right\rangle=C_{t}(f ; h)=\left\langle\delta_{f} \mid \delta_{h}\right\rangle \text { for all } f, h \in \mathcal{F}_{t} .
$$

Therefore, $C_{t}$ becomes the reproducing kernel of $\mathfrak{X}_{t}$. Now, due to $\mathcal{F}_{r} \subseteq \mathcal{F}_{t}$ for all $r<t$ and the projectivity (4.7) of $C$ we have isometric embeddings $\mathcal{F}_{r} \mathcal{A} \subseteq$ $\mathcal{F}_{t} \mathcal{A}$ for the semi-Hilbert $\mathcal{A}$-modules and thus the isometries $F_{t}^{\dagger}(r): \mathfrak{D}_{r} \hookrightarrow \mathfrak{D}_{t}$ on their quotients, which is extended by continuity to Hilbert $\mathcal{A}$-modules $\mathfrak{X}_{t}$. Moreover, we have that

$$
x_{t}(f)=F_{t}^{\dagger}(s) x_{s}(f) \quad \text { for any } f \in \mathcal{F}_{s}
$$


since for each function $f \in \mathcal{F}_{s} \subseteq \mathcal{F}_{t}, \delta_{f}$ as a function on $\mathcal{F}_{t}$ coincides with $\delta_{f}$ as a function on $\mathcal{F}_{s}$. It is obvious from the construction that $F=\left\{F_{r}(t): r<t \in T\right\}$ is a hemigroup of co-isometries $F_{r}(t): \mathfrak{X}_{t} \rightarrow \mathfrak{X}_{r}(r<t)$.

We note that the left multiplication operator $\lambda_{t}(a): h \mapsto a_{t} \cdot h$, given on $h \in \mathcal{F}_{t}$ by the function $a_{t}=a \delta_{t}$ for $a \in \mathcal{A}$ with the single point support $\tau\left(a_{t}\right)=\{t\}$ as

$$
\lambda_{t}(a): \mathbf{b}=\left(b_{1}, \ldots, b_{n}, b\right) \mapsto a_{t} \cdot \mathbf{b}=\left(b_{1}, \ldots, b_{n}, a b\right)
$$

on $b=h(t)$, is adjointable on $\mathfrak{D}_{t}$. Therefore, there exists a *-representation $\pi_{t}: \mathcal{A} \rightarrow \mathcal{L}_{\mathcal{A}}\left(\mathfrak{X}_{t}\right)$ extending the left multiplication $\pi_{t}(a) x_{t}(h)=x_{t}\left(a_{t} \cdot h\right)$ such that $\pi_{t}(a)^{\dagger}=\pi_{t}\left(a^{*}\right)$ due to

$$
\left\langle x_{t}(f) \mid \pi_{t}(a) x_{t}(h)\right\rangle=\left\langle\pi_{t}\left(a^{*}\right) x_{t}(f) \mid x_{t}(h)\right\rangle .
$$

This representation is obviously unital, $\pi_{t}(1)=I_{t}$, due to $1_{t} \cdot \mathbf{b}=\mathbf{b}$ as $1_{t}=\delta_{t}$.

Since $\mathcal{F}_{t} \subseteq \mathcal{F}_{t^{\prime}}$ for any $t^{\prime}>t$, by (4.9) and (4.10), the backward hemigroup $F=\left\{F_{s}(t): s<t \in T\right\}$ of co-isometries $F_{s}(t): \mathfrak{X}_{t} \rightarrow \mathfrak{X}_{s}$ with $e_{t}=F_{t}^{\dagger}(0) 1$ defines the reconstruction (4.4) as the kernel

$$
\kappa_{0}^{\tau}\left(\mathbf{a}^{*}, \mathbf{a}\right)=\left\langle x_{t}(f) \mid x_{t}(f)\right\rangle \equiv\left|x_{t}(f)\right|^{2}, \quad \forall f: \tau(f) \subseteq \tau
$$

with $f(0)=1, f\left(t_{i}\right)=a_{i}$ for $i=1, \ldots, n$ and $f(t t)=a$, where

$$
x_{t}(f)=\pi_{t}(a) F_{t}^{\dagger}\left(t_{n}\right) \pi_{t_{n}}\left(b_{n}\right) F_{t_{n}}^{\dagger}\left(t_{n-1}\right) \cdots \pi_{t_{1}}\left(b_{1}\right) F_{t_{1}}^{\dagger}(0) 1 .
$$

The Markovianity (4.3) simply follows from the recurrent relation for

$$
x_{t}(f)=\pi_{t}(a) F_{t}^{\dagger}\left(t_{n}\right) x_{t_{n}}\left(f_{n}\right) \equiv x_{t}\left(a_{t} \cdot f_{n}\right),
$$

where $\tau\left(f_{n}\right) \subseteq \tau \backslash\{t\}$ such that $f_{n}(t)=1$ for $t=\max \tau$. We have that

$$
\begin{aligned}
\left\langle x_{t}(f) \mid x_{t}(h)\right\rangle & =\left\langle F_{t}^{\dagger}\left(t_{n}\right) x_{t_{n}}\left(f_{n}\right) \mid \pi_{t}\left(a^{*} b\right) F_{t}^{\dagger}\left(t_{n}\right) x_{t_{n}}\left(h_{n}\right)\right\rangle \\
& =\left\langle x_{t_{n}}\left(f_{n}\right) \mid \pi_{t_{n}}\left(\phi_{t-t_{n}}\left(a^{*} b\right)\right) x_{t_{n}}\left(h_{n}\right)\right\rangle
\end{aligned}
$$

for all $f_{n} \in \mathcal{F}_{t_{n}}$, where the latter identity giving the strong Markovianity is due to

$$
\kappa_{0}^{\tau}\left(\mathbf{a}_{n}^{*} \cdot a_{t}^{*}, b_{t} \cdot \mathbf{b}_{n}\right)=\kappa_{0}^{\tau_{n}}\left(\mathbf{a}_{n}^{*},\left[\phi_{t-t_{n}}\left(a^{*} b\right)\right]_{t_{n}} \mathbf{b}_{n}\right)
$$

for $\tau_{n}=\tau \backslash\{t\}$ with $\max \tau_{n}=t_{n}$.

A time indexed family $\pi=\left\{\pi_{t}: t \in T\right\}$ of unital $*$-morphisms $\pi_{t}$ from $\mathcal{A}$ into $\mathcal{L}_{\mathcal{A}}\left(\mathfrak{X}_{t}\right)$ with the increasing identity $I_{t}=\pi_{t}(1)$ on $\mathfrak{X}_{t}$ is called a monotone quantum stochastic process in the strong sense of Belavkin [2,3]. Hence, the family $\pi=\left\{\pi_{t}: t \in T\right\}$, obtained in Theorem 4.1, of $*$-representations $\pi_{t}$ of $\mathcal{A}$ on $\mathfrak{X}_{t}$ is a monotone quantum stochastic process.

Remark 4.2. The result of Theorem 4.1 can be extended to the case of an arbitrary totally ordered set $T$ with a minimal element $\emptyset=\min T$ and considered as a dynamical Hilbert $C^{*}$-module representation of the Stinespring's one. 


\section{Covariant quantum dynamical semigroups}

Let $T=[0, \infty)$ and $S$ be a symmetry semigroup. Let $\mathcal{A}$ be a unital $C^{*}$ algebra with the unit element 1 and let $\left(u_{s}\right):=\left\{u_{s} \in \mathcal{U}(\mathcal{A}): s \in S\right\}$ be a representation of $S$ in the unitary group $\mathcal{U}(\mathcal{A})$. Let $(\mathcal{A}, S, \theta)$ be a $C^{*}$-dynamical system. A quantum dynamical semigroup $\left\{\phi_{t}: t \geq 0\right\}$ on $\mathcal{A}$ is $(\theta, u)$-covariant if $\phi_{t}$ is $(\theta, u)$-covariant for any $t \geq 0$, that is,

$$
\phi_{t}\left(\theta_{s}(a)\right)=u_{s} \phi_{t}(a) u_{s}^{*} \quad \text { for all } a \in \mathcal{A}, s \in S, t \in T .
$$

We shall assume that for any $s \in S, \theta_{s}: \mathcal{A} \rightarrow \mathcal{A}$ is unitarily implemented as

$$
\theta_{s}(a)=u_{s} a u_{s}^{*}, \quad(a \in \mathcal{A}) .
$$

A covariant dynamical representation of $(\mathcal{A}, S, \theta)$ is a triple $(\pi, V, \mathfrak{X})$, where $\pi=\left(\pi_{t}\right)$ is a stochastic process given by unital *-representations $\pi_{t}: \mathcal{A} \rightarrow$ $\mathcal{L}_{\mathcal{A}}\left(\mathfrak{X}_{t}\right)$ on the increasing Hilbert $\mathcal{A}$-module system $\mathfrak{X}=\left(\mathfrak{X}_{t}\right)$ with $\pi_{0}=\mathrm{id}_{\mathcal{A}}$ on $\mathfrak{X}_{0}=\mathcal{A}$, the hemigroup $F$ of the co-isometries $F_{r}(t): \mathfrak{X}_{t} \rightarrow \mathfrak{X}_{r}, t>r \in T$, $F_{t}^{\dagger}(0) 1=e_{t}$ and $V=\left\{V_{(\cdot)}(t): t \in T\right\}$ is a family of semigroups $\left\{V_{s}(t): s \in S\right\}$ indexed by $t \in T$ :

$$
V_{r}(t) V_{s}(t)=V_{r \cdot s}(t), \quad(r, s \in S)
$$

of an isometric representation $V(t): S \rightarrow \mathcal{B}_{\mathcal{A}}\left(\mathfrak{X}_{t}\right)$ with an initial unitary $V_{s}(0)=u_{s}$, satisfying the cocycle and consistency conditions

$$
\begin{gathered}
\pi_{t}\left(\theta_{s}(a)\right) V_{s}(t)=V_{s}(t) \pi_{t}(a), \quad(a \in \mathcal{A}, s \in S, t \in T), \\
F_{t_{0}}(t) V_{s}(t)=V_{s}\left(t_{0}\right) F_{t_{0}}(t), \quad\left(s \in S, t>t_{0} \in T\right) .
\end{gathered}
$$

Now, we are going to study a Hilbert $C^{*}$-module version for the stationary case of the covariant reconstruction theorem for the monotone Markov process indexed by a totally ordered set $T$ as formulated for the Hilbert space case and the general set $T[2]$.

Theorem 5.1. Let $(\mathcal{A}, S, \theta)$ be a $C^{*}$-dynamical system. If $\phi=\left\{\phi_{t}: t \in T\right\}$ is $a(\theta, u)$-covariant dynamical semigroup over the unital $C^{*}$-algebra $\mathcal{A}$ with unit 1 , then there exist

(i) an increasing Hilbert $\mathcal{A}$-module system $\mathfrak{X}=\left(\mathfrak{X}_{t}\right)$,

(ii) a stochastic process $\pi=\left(\pi_{t}\right)$ given by unital $*$-representations $\pi_{t}: \mathcal{A} \rightarrow$ $\mathcal{L}_{\mathcal{A}}\left(\mathfrak{X}_{t}\right)$,

(iii) a hemigroup $F=\left\{F_{r}(t): r<t \in T\right\}$ of co-isometries $F_{r}(t): \mathfrak{X}_{t} \rightarrow \mathfrak{X}_{r}$,

(iv) a family $V=\left\{V_{(\cdot)}(t): t \in T\right\}$ of semigroups $\left\{V_{s}(t): s \in S\right\}$ of unitaries $V_{s}(t) \in \mathcal{L}_{\mathcal{A}}\left(\mathfrak{X}_{t}\right)$ satisfying $(5.3)$

such that the cocycle and consistency conditions (5.4) and (5.5) hold.

Proof. In Theorem 4.1, the family $\mathfrak{X}=\left(\mathfrak{X}_{t}\right)$ of Hilbert $\mathcal{A}$-modules, a quantum stochastic process $\pi=\left(\pi_{t}\right)$ and a hemigroup $F=\left\{F_{r}(t): r<t \in T\right\}$ are already constructed. 
Using the notations in Theorem 4.1, for each $s \in S$, we define the action $\Theta_{s}$ onto the set $\mathcal{F}$ of finitely supported $\mathcal{A}$-valued functions $f \in \mathcal{F}$ by $\Theta_{s}(f)(r)=$ $\theta_{s}(f(r))$ for any $r \in T$. If $\tau=\operatorname{supp}(h)$ is a chain $\left\{t_{1}, \ldots, t_{n}, t\right\}$, the support of $\Theta_{s}(h)$ is also the chain $\tau=\left\{t_{1}, \ldots, t_{n}, t\right\}$. This determines a linear operator

$$
\mathcal{F}_{t} \mathcal{A} \ni g \longrightarrow \sum_{i} \Theta_{s}\left(f_{i}\right) \theta_{s}\left(a_{i}\right) u_{s} \in \mathcal{F}_{t} \mathcal{A}
$$

on each space $\mathcal{F}_{t} \mathcal{A}$ of all finite $\mathcal{A}$-combinations $g=\sum_{i} f_{i} a_{i}$ of $f_{i} \in \mathcal{F}_{t}$. On the other hand, it follows from (5.1), (5.2) and (4.6) that

$$
\kappa_{0}^{\tau}\left(\mathbf{a}_{s}^{*}, \mathbf{b}_{s}\right)=u_{s} \kappa_{0}^{\tau}\left(\mathbf{a}^{*}, \mathbf{b}\right) u_{s}^{*}, \quad\left(\mathbf{a}, \mathbf{b} \in \mathcal{A}^{|\tau|}\right)
$$

where $\mathbf{a}_{s}=\left(a_{1 s}, \ldots, a_{n s}, a_{s}\right)$ with $a_{i_{s}}=\theta_{s}\left(a_{i}\right), \mathbf{b}_{s}=\left(b_{1 s}, \ldots, b_{n_{s}}, b_{s}\right)$ with $b_{i s}=\theta_{s}\left(b_{i}\right)$ and $\tau=\left\{t_{1}, \ldots, t_{n}, t\right\}$. Therefore, due to (5.7) the operator given as in (5.6) gives an isometry

$$
V_{s}(t) h=\sum_{i} \Theta_{s}\left(f_{i}\right) \theta_{s}\left(a_{i}\right) u_{s}+\mathcal{N}_{t}, \quad \forall h=g+\mathcal{N}_{t} \in \mathfrak{D}_{t}
$$

on the pre-Hilbert $\mathcal{A}$-module $\mathfrak{D}_{t}$ in Theorem 4.1 , since

$$
\left\langle V_{s}(t) h \mid V_{s}(t) h\right\rangle=\sum_{i, k} a_{i}^{*} u_{s}^{*} \theta_{s}\left(\kappa_{0}^{\tau}\left(\mathbf{b}_{i}^{*}, \mathbf{b}_{k}\right)\right) u_{s} a_{k}=\langle h \mid h\rangle, \quad \forall h \in \mathfrak{D}_{t}
$$

due to the covariance (5.7) of the kernels (4.6) constructed from the covariant dynamical semigroup $\phi$. Therefore, $V_{s}(t)$ can be extended, by continuity, to $\mathfrak{X}_{t}$ as an isometry into $\mathfrak{X}_{t}$ for every $s \in S$ and each fixed $t \in T$. Moreover, these isometries have the $\mathcal{A}$-modular property since they commute with right multiplications $R_{a}$ by $a \in \mathcal{A}$ on $\mathfrak{X}_{t}$ as

$$
R_{a} V_{s}(t) h=\sum_{i} \Theta_{s}\left(f_{i}\right) \theta_{s}\left(a_{i}\right) u_{s} a+\mathcal{N}_{t}=V_{s}(t) R_{a} h
$$

for all $h \in \mathfrak{D}_{t}$ and $a \in \mathcal{A}$. Thus, we have that $V_{s}(t) \in \mathcal{B}_{\mathcal{A}}\left(\mathfrak{X}_{t}\right)$. Consider an action $v$ of $S$ on $\mathcal{A}$ by a semigroup of endomorphisms $s \mapsto v_{s} \in \operatorname{End}(\mathcal{A})$ such that for each $s \in S, v_{s}(a)=u_{s}^{*} a u_{s}$ for any $a \in \mathcal{A}$, and an operator $W_{s}(t)$ defined on $\mathfrak{D}_{t}$ by

$$
W_{s}(t) h=\sum_{i} \Upsilon_{s}\left(f_{i}\right) v_{s}\left(a_{i}\right) u_{s}^{*}+\mathcal{N}_{t}, \quad\left(h=\sum_{i} f_{i} a_{i}+\mathcal{N}_{t} \in \mathfrak{D}_{t}\right),
$$

where $\Upsilon_{s}(s \in S)$ is the action onto the set $\mathcal{F}$ of finitely supported $\mathcal{A}$-valued functions defined by $\Upsilon_{s}(f)(r)=v_{s}(f(r))$ for $f \in \mathcal{F}$ and $r \in T$. Then, by similar arguments used for $V_{s}(t)$, we can easily see that $W_{s}(t)$ is an isometry on $\mathfrak{D}_{t}$ and its extension to $\mathfrak{X}_{t}$ is the adjoint of $V_{s}(t)$. Therefore, $V_{s}(t) \in \mathcal{L}_{\mathcal{A}}\left(\mathfrak{X}_{t}\right)$ is unitary. The semigroup condition (5.3) for $V$ is obvious by the definition of $V$. Also, we can see that the cocycle conditions (5.4) and (5.5) by the arguments similar to [3]. 
Remark 5.2. In [10], Goswami and Sinha constructed Evans-Hudson dilations of a uniformly continuous quantum dynamical semigroup on a von Neumann algebra and they extended to unital separable $C^{*}$-algebras. Chakraborty, Goswami and Sinha [6] obtained covariant dilations for uniformly continuous semigroups. That is, they proved that every uniformly continuous quantum-dynamical semigroup on a separable $C^{*}$-algebra or a von Neumann algebra which is covariant under a compact group action admits a covariant Evans-Hudson dilation in some Fock space, which is the Hilbert space analogue of Theorem 5.1. To get covariant dilations for uniformly continuous semigroups, they used Hilbert module techniques, which are quite close to our techniques. However, they did not consider a Hilbert module framework for Bhat-Parthasarathy-Belavkin type dilation and the stationarity of a quantum stochastic process which are proved in this section. For a general setting, we did not restrict to compact groups.

For each fixed $t \in T$ and $a \in \mathcal{A}$, we consider the quantum stochastic process $\left\{\pi_{t}\left(\theta_{s}(a)\right): s \in S\right\}$ and its correlation function

$$
C_{r, s}^{(t)}(a)=\left\langle\pi_{t}\left(\theta_{s}(a)\right) e_{t} \mid \pi_{t}\left(\theta_{s}(a)\right) e_{t}\right\rangle .
$$

Then we get the following theorem for the stationarity of the quantum stochastic process $\pi$ given as in Theorem 5.1.

Theorem 5.3. The quantum stochastic process $\left\{\pi_{t}\left(\theta_{s}(a)\right): s \in S\right\}$ is $u$ stationary in the sense that

$$
C_{v \cdot r, v \cdot s}^{(t)}(a)=u_{v} C_{r, s}^{(t)}(a) u_{v}^{*}, \quad \forall r, s, v \in S .
$$

Proof. For any $r, s, v \in S$, we obtain that

$$
\begin{aligned}
C_{v \cdot r, v \cdot s}^{(t)}(a) & =\left\langle\pi\left(\theta_{v \cdot r}(a)\right) e_{t} \mid \pi\left(\theta_{v \cdot s}(a)\right) e_{t}\right\rangle \\
& =u_{v} C_{r, s}^{(t)}(a) u_{v}^{*},
\end{aligned}
$$

which implies the equation (5.8).

\section{References}

[1] L. Accardi, A. Frigerio, and J. T. Lewis, Quantum stochastic processes, Publ. Res. Inst. Math. Sci. 18 (1982), no. 1, 97-133.

[2] V. P. Belavkin, A reconstruction theorem for a quantum random field, Uspekhi Mat. Nauk 39 (1984), no. 2, 137-138.

[3] _ Reconstruction theorem for a quantum stochastic process, Theor. Math. Phys. 62 (1985), 275-289.

[4] B. V. Bhat and K. R. Parthasarathy, Markov dilations of nonconservative dynamical semigroups and a quantum boundary theory, Ann. Inst. H. Poincaré Probab. Statist. 31 (1995), no. 4, 601-651.

[5] B. V. Bhat and M. Skeide, Tensor product systems of Hilbert modules and dilations of completely positive semigroups, Infin. Dimens. Anal. Quantum Probab. Relat. Top. 3 (2000), no. $4,519-575$. 
[6] P. S. Chakraborty, D. Goswami, and K. B. Sinha, A covariant quantum stochastic dilation theory, Stochastics in finite and infinite dimensions, 89-99, Trends Math., Birkhäuser, Boston, 2001.

[7] E. Christensen and D. Evans, Cohomology of operator algebras and quantum dynamical semigroups, J. London Math. Soc. (2) 20 (1979), no. 2, 358-368.

[8] E. B. Davies, Quantum stochastic processes, Comm. Math. Phys. 15 (1969), 277-304.

[9] _ Markovian master equations, Comm. Math. Phys. 39 (1974), 91-110.

[10] D. Goswami and K. B. Sinha, Hilbert modules and stochastic dilation of a quantum dynamical semigroup on a von Neumann algebra, Comm. Math. Phys. 205 (1999), no. 2, 377-403.

[11] J. Heo, Completely multi-positive linear maps and representations on Hilbert $C^{*}$ modules, J. Operator Theory 41 (1999), no. 1, 3-22.

[12] _ Hilbert $C^{*}$-module representation on Hagerup tensor products and group systems, Publ. Res. Inst. Math. Sci. 35 (1999), no. 5, 757-768.

[13] J. Heo, V. P. Belavkin, and U. C. Ji, Monotone quantum stochastic processes and covariant dynamical hemigroups, J. Func. Anal. 261 (2011), 3345-3365.

[14] E. Lance, Hilbert $C^{*}$-modules, Cambridge University Press, 1995.

[15] G. Lindblad, On the generators of quantum dynamical semigroups, Comm. Math. Phys. 48 (1976), no. 2, 119-130.

[16] _ Non-Markovian quantum stochastic processes and their entropy, Comm. Math. Phys. 65 (1979), no. 3, 281-294.

[17] K. Parthasarathy, A continuous time version of Stinespring's theorem on completely positive maps, Quantum probability and applications, V (Heidelberg, 1988), 296-300, Lecture Notes in Math., 1442, Springer, Berlin, 1990.

[18] W. Paschke, Inner product modules over $B^{*}$-algebras, Trans. Amer. Math. Soc. 182 (1973), 443-468.

JAESEONG HEO

Department of MATHEMATiCs

Research Institute FOr Natural Sciences

HANYANG UNIVERSITY

SEOUl 133-791, KoreA

E-mail address: hjs@hanyang.ac.kr

ViaChESLAV P. BELAVKIN

Mathematics Department

UNIVERSITY OF NOTTINGHAM

University PARK, Nottingham NG72RD, UK

E-mail address: vpb@maths.nott.ac.uk

UN Cig Ji

Department of Mathematics

Research Institute of Mathematical Finance

Chungbuk National University

Cheonguu 361-763, Korea

E-mail address: uncigji@chungbuk.ac.kr 